\title{
MATÉRIEL ET TECHNIQUE D'INSÉMINATION ARTIFICIELLE UTILISESS EN FRANCE POUR L'ESPÈCE PORCINE RÉSULTATS OBTENUS
}

\author{
F. DU MESNIL DU BUISSON. \\ avec la collaboration technique de A. LocatedLI, \\ J. B. Meurot et J. Goussopoulos. \\ Station de Recherches de Physiologie animale, \\ Centre national de Recherches zootechniques, Jouy-en-Josas (Seine et Oise).
}

\section{SOMMAIRE}

La technique d'insémination décrite, qui a été utilisé dans la pratique sur to 000 truies est fondée sur le principe d'écoulement du sperme par gravité. La durée de l'insémination est fonction des contractions du col et de l'utérus. Pour $250 \mathrm{~cm}^{3}$ de sperme dilué, elle est de 4 à 7 minutes dans $52 \mathrm{p}$. Ioo des cas. Les inséminations les plus rapides sont faites dans la première moitié de l'œstrus ou sur des truies parfaitement calmes.

Le matériel utilisé comprend une sonde en caoutchouc rigide de $20 \mathrm{~mm}$ de diamètre (entourée à son extrémité par un ballonnet formant un manchon de $35 \mathrm{~mm}$ de diamètre sur $8 \mathrm{~cm}$ de longueur), un tube de polythène de I mètre et un récipient du type "flacon à plasma " qui est tenu à $40 \mathrm{~cm}$ au dessus du dos de la truie. La sonde est attachée par un lien élastique à la queue de l'animal.

Le sperme est conservé prédilué à $15^{\circ} \mathrm{C}$ en ampoules scellées en présence de gaz carbonique suivant une technique décrite antérieurement par L. DAUZIER et nous-mêmes. Il est dilué une deuxième fois extemporanément.

Les résultats suivants sont donnés en pourcentage de mise-bas contrôlées après une seule insémination. Sur 4438 inséminations, le pourcentage global de réussites est de 5I,3 p. Ioo. Il n'y a pas de différence significative entre les résultats enregistrés avec du sperme utilisé le jour même de la collecte $\left(\mathrm{J}_{0}\right)$ ou le lendemain $\left(\mathrm{J}_{1}\right)$. On a obtenu $46,7 \mathrm{p}$. Ioo le jour $\mathrm{J}_{2}$ sur $662 \mathrm{I}$. A., et 46,7 p. 1oo le jour $\mathrm{J}_{3}$ sur $107 \mathrm{I}$. A. et pour les jours suivants $\mathrm{J}_{4}, \mathrm{~J}_{5}$ et $\mathrm{J}_{6} 45,4 \mathrm{p}$. I 00 sur un total de $33 \mathrm{I}$. A.

La classification des truies, en tenant compte de leur comportement au moment de l'I. A., fait apparaître des différences importantes dans les pourcentages de réussite : ainsi chez 87 I truies « très calmes " le pourcentage s'élève à 63,8 .

Le nombre de porcelets par portée est normal, pour les races utilisées soit, en moyenne, 8,72 pour la race normande et 9,37 pour la race Large White.

L'avancement des recherches sur la physiologie de la reproduction du Porc et sur la technologie du sperme de Verrat ont encouragé les essais d'insémination artificielle. Leur succès laisse prévoir une généralisation prochaine de l'insémination dans l'espèce porcine; il nous a donc semblé utile de décrire aussi bien le matériel que la technique d'insémination proprement dite qui ont été utilisés dans la pratique en France, depuis près de trois ans, sur Io ooo Truies. 


\section{TECHNIQUE ET MATÉRIEL, UTILISÉS A I,'ÉTRANGER}

Le matériel d'insémination comprend deux éléments essentiels : une sonde et un récipient dans lequel se trouve le sperme.

Ito, Nrwa et Kudo (I948) les associent en un seul instrument très original. En effet la sonde est constituée par un cylindre creux (diam. $20 \mathrm{~mm}$ ) dans lequel se meut un piston. Le corps même de cette seringue est introduit profondément dans le vagin et le sperme est poussé par la pression exercée par le piston. L'inconvénient principal de cet instrument, que nous avons utilisé pour près de 500 inséminations, est sa rigidité ; même s'il est pratiquement incassable (Lucoflex), il risque de blesser la truie quand elle s'agite.

Les premières sondes proposées étaient également rigides puisque constituées par un tube de verre légèrement coudé à son extrémité (Milovaxov I934, Goetze I949, cité par HoF, I956). Les sondes actuellement utilisées sont, au contraire, plus ou moins souples.

Nrwa (I958) lui-même, emploie maintenant une sonde en caoutchouc. Elle est ouverte, non pas à son extrémité, mais par des pores situés à la base d'un apex qui prolonge une olive en caoutchouc rigide, placée là pour empêcher le reflux.

KVASNiski et Konyukova (I958) ont conçu une sonde du même type mais dont l'olive est remplacée par une boule plus grosse, en caoutchouc mousse ; cette sonde a une gaine métallique.

POLGE (I956) a décrit une sonde rectiligne en caoutchouc assez rigide; son fort diamètre lui permet d'adhérer plus ou moins étroitement à la paroi du vagin.

Enfin le système du ballonnet a été très habilement aménagé sur la sonde en plastique de AAMDAL (I957) : à 2 centimètres de son extrémité se trouve un disque amovible qui, gonflé, se présente comme un torre bouchant la lumière vaginale. Cette sonde a été utilisée avec succès pour des milliers d'inséminations.

La légère pression nécessaire pour faire pénétrer le sperme dans les cornes utérines peut être obtenue de différentes manières : ou bien on peut créer cette pression dans le récipient qui contient le sperme, ou bien on peut provoquer son écoulement par gravité en plaçant le flacon de sperme à une certaine hauteur au dessus du point de pénétration. Plusieurs types d'appareils ont donc été conçus.

Les Japonais aboutent la sonde à une seringue de verre de Ioo cc. AAmdal, se sert d'une petite bouteille en matière plastique très souple de $50 \mathrm{cc}$., contenant exactement la dose de sperme dilué nécessaire pour une truie. Pour l'insémination l'opérateur comprime la bouteille avec sa main et la vide complètement. Cette méthode offre de grandes commodités pour le transport en même temps que de sérieuses garanties d'hygiène. Elle présente cependant l'inconvénient de ne permettre le réglage de la pression à exercer qu'en fonction du début de reflux. De plus, comme la précédente et la suivante, elle oblige l'inséminateur à suivre la truie chaque fois qu'elle se déplace.

Avec le flacon hermétique de verre dans lequel PoLGe crée une pression avec une poire, on peut craindre, si la sonde se déplace, de voir le flacon se vider en dehors du cervix.

Une pression plus facilement contrôlable peut être obtenue par une dénivellation entre l'extrémité de la sonde et le récipient où se trouve le sperme. C'est 
le système du bock, classique pour les lavements ou les injections vaginales, qu'ont utilisé Mrlovanov et Goetze. Kvasnisky (I959) utilise un dispositif de ce type avec deux récipients et une possibilité d'injecter de l'air grâce à une poire de Richardson. L'inconvénient de cet appareillage est sa complexité.

\section{MATÉRIEL UTILISÉ PAR NOUS}

La sonde est en caoutchouc assez dur; son diamètre $(20 \mathrm{~mm})$ et l'épaisseur de ses parois $(6 \mathrm{~mm})$ contribuent à lui donner de la rigidité. Longue de $45 \mathrm{~cm}$, elle peut pénétrer jusqu'au col. Son extrémité, tronc conique sur une longueur de $35 \mathrm{~mm}$, est percée par un trou de $4 \mathrm{~mm}$ de diamètre. Un ballonnet gonflable et amovible, placé à 5 ou $6 \mathrm{~cm}$ de l'extrémité, enserre la sonde sur une longueur de $8 \mathrm{~cm}$, et forme un manchon de $35 \mathrm{~mm}$ de diamètre. Pour rendre le ballonnet moins fragile, on l'a moulé en feuille anglaise assez épaisse en ayant soin qu'il ne présente aucune tension quand il est dégonflé.

Le récipient est un $甘$ lacon à plasma classique gradué, en verre " asolvex ". On l'emploie avec le bouchon de caoutchouc, la capsule métallique à vis et l'étrier en plastique prévus pour l'écoulement goutte à goutte du plasma. Un tube plonge dans le fond pour permettre l'entrée de 1'air. Ce matériel de grande série est bon marché.

Un tube de I mètre en polythène (diamétre extérieur : $4 \mathrm{~mm}$ ) de qualité médicale est placé entre le flacon et la sonde. Il est relié au bouchon et à l'entrée de la sonde par un embout effilé en verre épais ou en Lucoflex (plastique rigide). L'emploi d'un tube calibré dans le circuit permet de limiter le débit.

Ce matériel d'insémination est complété par un lien élastique et une pince (type pince à hémostase) nécessaire pour maintenir la sonde en place dans le vagin en la rendant solidaire de la queue de la truie.

\section{TECHNIQUE D'INSÉMINATION ARTIFICIELLE}

Dans la pratique de l'insémination artificielle, la seule technique de conservation du sperme utilisée actuellement en France est celle que nous avons décrite avec L. DAUZIER en I958.

Le dilueur de Van Demark et Sharma (I957) sans jaune d'œuf ( ${ }^{1}$ ) peut être préparé à l'avance et conservé une semaine, à condition que les antibiotiques ne soient additionnés au dilueur qu'au début de la journée d’utilisation. Pour éviter un jaunissement de la solution, on a intérêt à chauffer très doucement entre $40^{\circ} \mathrm{C}$ et $70^{\circ} \mathrm{C}$.

Dès que le dilueur est saturé de gaz carbonique à la température de $30^{\circ} \mathrm{C}$, on incorpore les antibiotiques, puis on mélange le dilueur et le sperme filtré, volume à volume.

Le sperme ainsi dilué est réparti en ampoules représentant chacune la dose

(1) Note: Compositon du dilueur pour r litre d'eau distillée : citrate trisodique I $\mathrm{H}_{2} \mathrm{O}, 24,280 \mathrm{gr}$; bicarbonate de sodium, 2, I $\mathrm{gr}$; chlorure de potassium, $0,4 \mathrm{gr}$; glucose anhydre, $3,0 \mathrm{gr}$; para-amino-phényl-sulfamide, $3, \circ \mathrm{gr}$; barbotage du $\mathrm{CO}_{2}$ pendant to minutes $(\mathrm{pH} 6,3-6,4)$.

Après barbotage, pénicilline I $000000 \mathrm{U}$. I. et streptomycine I gr. 
de spermatozoïdes nécessaires pour une insémination artificielle (ampoule de 30-40 ou $5^{\circ}$ ce suivant la concentration du sperme).

Les ampoules sont garnies en trempant une extrémité dans le récipient pendant que l'autre est reliée à une trompe à eau qui crée une légère dépression ; après scellement fait au chalumeau (butagaz, lance "Express " de type Bijoutier, sans oxygène),

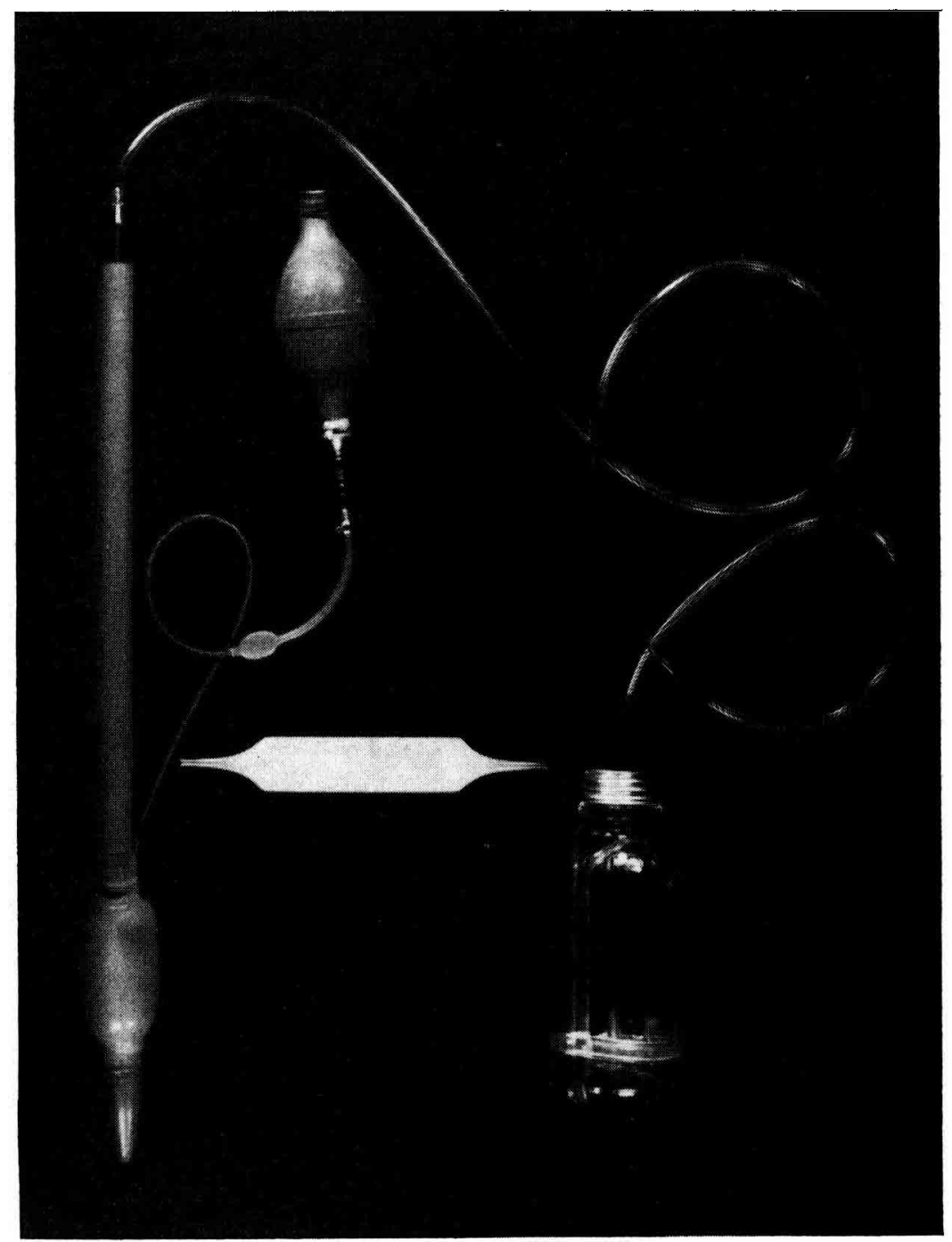

FIG.I . - Le matériel d'insémination artificielle utilisé en France.

elles sont étiquetées et laissées à la température ambiante jusqu'à ce que leur contenu se trouve lui aussi à cette température. Enfin on les immerge dans un bac d'eau à $I 5^{\circ} \mathrm{C}$ où elles sont stockées.

Pour obtenir une température absolument constante de $15^{\circ} \mathrm{C}$, on place le bac dans un réfrigérateur réglé à une température inférieure à $15^{\circ} \mathrm{C}$ et on maintient à 
I $5^{\circ}$ la température de l'eau au moyen d'une résistance de 500 watts reliée à un thermomètre de contact au $I_{1} I O^{\mathbf{e}}$ de degré $\mathrm{C}$. Ensuite les ampoules sont transportées par les inséminateurs dans de grandes bouteilles thermos contenant de $1^{\prime}$ eau à $I 5^{\circ}$. Pour les expéditions à longue distance nous avons utilisé des boîtes en matériaux très légers (Frigolit) dans lesquelles les ampoules se trouvaient au contact de flacons en plastique contenant de l'acide acétique congelé (température de cristallisation I6, $6^{\circ} \mathrm{C}$; chaleur latente, 43,2 calories).

Le dilueur qui servira à la dilution extemporanée est le même que celui qui a été employé pour la première dilution, mais il ne contient pas de gaz carbonique ; il est transporté dans des thermos à la température de $30^{\circ} \mathrm{C}$, ceci afin de permettre un réchauffement partiel du sperme avant l'insémination.

Au moment même de l'insémination, après avoir vidé le contenu de l'ampoule dans le flacon à plasma, l'inséminateur y verse doucement le dilueur jusqu'à la graduation 250 .

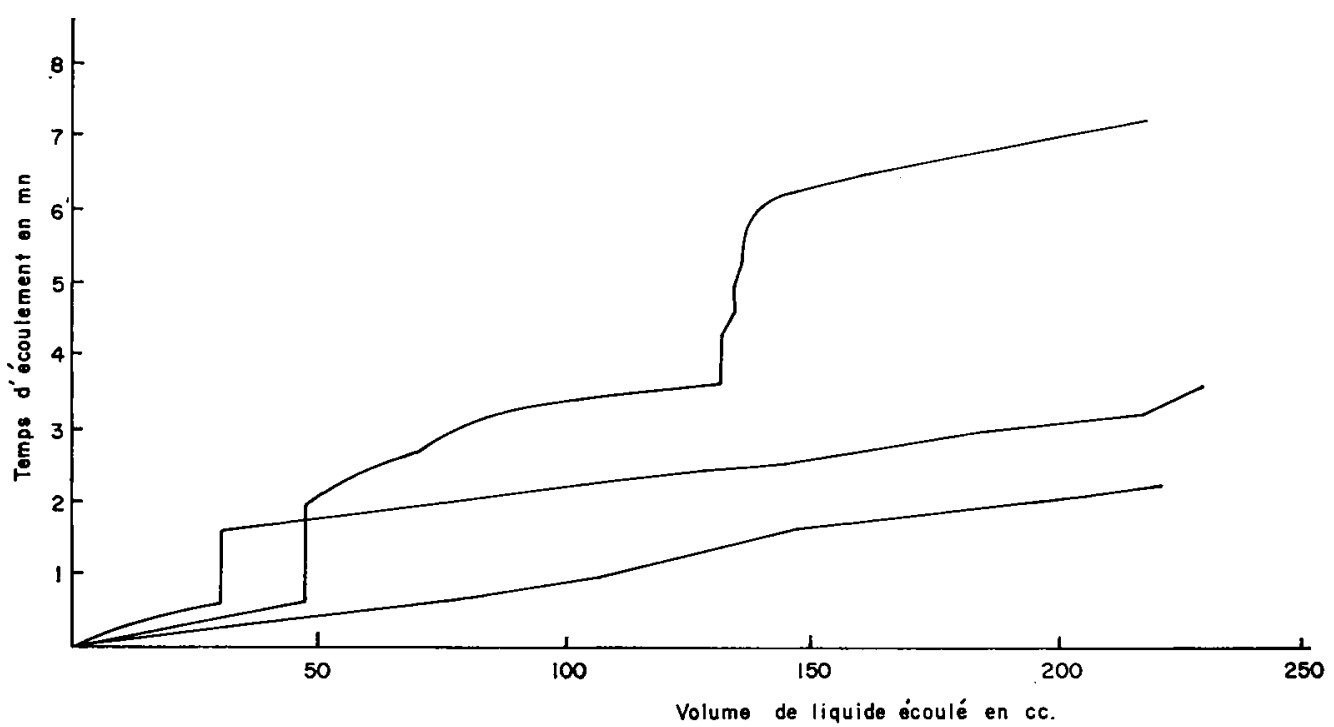

FIG. 2. - Courbes typiques d'enregistrement de l'écoulement du sperme pour trois trites inseminées 30 heures après le début de l'asinus.

L'opérateur introduit ensuite dans le vagin la sonde après l'avoir mouillée avec un peu de dilueur ; lorsqu'elle bute sur les premières tubérosités du col, il la recule d'un demi centimètre afin de dégager l'ouverture. Il fixe alors la sonde à la base de la queue au moyen de l'élastique et de la pince, puis gonfle très légèrement le ballon; un excès de gonflage est nuisible, car il provoque une réaction de défense de la truie.

I a sonde est ensuite reliée par le tube en plastique au flacon que l'inséminateur tiendra à la hauteur de sa poitrine pendant toute l'insémination.

Ces opérations ne nécessitent aucune contention de la truie, pas plus que l'emploi d'une cage, contrairement aux techniques indiquées par BonadonNa (I940) ou LIEBENBERG (I952) : elles sont réalisées sans isoler la truie de ses compagnes. Même lorsque la truie est agitée, la sonde reste normalement en place.

De même il est inutile de soulever l'arrière train de la truie avec un palan 
GoETzE, I949) pour obtenir un écoulement régulier du sperme. Quant à la pratique qui consiste à obstruer la vulve avec un morceau de coton en fin d'opération, elle est illusoire, car le sperme qui reflue dans le vagin a très peu de chance de pénétrer à nouveau dans l'utérus.

L'avantage de la technique d'insémination artificielle par gravité tient essentiellement à la possibilité d'utiliser une pression faible, insuffisante pour provoquer l'écoulement forcé du sperme et son reflux quand le col et l'utérus se contractent, mais telle cependant qu'elle assure son passage dans l'utérus pendant les phases de relaxation du col.

Au cours de l'insémination, la durée de l'opération, comme l'allure de la courbe d'écoulement, reflètent alors la réaction du tractus génital de la truie. Aussi les avons-nous étudiées sur un certain nombre de truies.

\section{DURÉE DE I'INSÉMINATION}

Une pression de $40 \mathrm{~cm}$ d'eau étant suffisante pour l'insémination, nous l'avons choisie pour enregistrer la vitesse d'écoulement. Nous avons utilisé pendant nos mesures un tube calibré (diamètre : $I, 33 \mathrm{~mm}$ ) permettant l'écoulement de $250 \mathrm{~cm}^{3}$ de sperme en I minute 40 secondes, c'est-à-dire un temps inférieur à la durée d'éjaculation d'un même volume pendant la collecte du sperme.

L'enregistrement des quantités de liquide écoulé en maintenant le niveau supérieur du liquide à hauteur constante, a permis, par lecture toutes les ro secondes, d'établir des courbes de vitesse de pénétration pendant l'insémination artificielle; trois sont donnés à titre d'exemple à la figure 2. Elles comprennent presque toujours un ou plusieurs paliers reliés par des lignes de pente voisines de celle correspondant à l'écoulement libre du tube calibré.

On a noté la durée totale de 1'écoulement dans plus de 250 cas : sur deux lots de 146 et I33 inséminations faites respectivement chez les éleveurs et en porcherie expérimentale, la durée moyenne pour 1'introduction de $250 \mathrm{cc}$ de sperme a été de 6 minutes 52 secondes et 5 minutes 57 secondes.

TABLEAU I

Chaleurs non testées (truies inséminées chez les éleveurs)

\begin{tabular}{|c|c|c|c|c|c|c|}
\hline \multirow{2}{*}{ Comportement } & \multicolumn{2}{|c|}{ Nullipares } & \multicolumn{2}{|c|}{ Truies ayant déja porté } & \multicolumn{2}{|c|}{ Toutes Truies } \\
\hline & $\begin{array}{l}\text { Tombre } \\
\text { d'IA }\end{array}$ & $\begin{array}{c}\text { Durée moyenne } \\
\text { de l'IA }\end{array}$ & $\begin{array}{l}\text { Nombre } \\
\text { d'IA }\end{array}$ & $\begin{array}{c}\text { Durée moyenne } \\
\text { de l'IA }\end{array}$ & $\begin{array}{l}\text { Nombre } \\
\text { d'IA }\end{array}$ & $\begin{array}{c}\text { Durée moyenne } \\
\text { de l'IA }\end{array}$ \\
\hline Très calme & 15 & $6 \mathrm{mn} 13 \mathrm{~s}$ & 55 & $6 \mathrm{mn} 20 \mathrm{~s}$ & 70 & $6 \mathrm{mn} 18 \mathrm{~s}$ \\
\hline Autre comportement. & 31 & $7 \mathrm{mn} 55 \mathrm{~s}$ & $4 \check{5}$ & $7 \mathrm{mn} 02 \mathrm{~s}$ & 76 & $7 \mathrm{mn} 23 \mathrm{~s}$ \\
\hline Tous comportements & 46 & $7 \mathrm{mn} 22 \mathrm{~s}$ & 100 & $6 \mathrm{mn} 39 \mathrm{~s}$ & 146 & $6 \mathrm{mn} 52 \mathrm{~s}$ \\
\hline
\end{tabular}

Sur le lot de I 46 inséminations (tableau I), 52 p. Ioo des inséminations ont une durée comprise entre 4 et 7 minutes et seulement I 2 p. Ioo prennent plus de ro minutes (jusqu'à I4 minutes) ; la vitesse de pénétration semble plus grande pour les truies qui ont déjà porté que pour les truies nullipares 6 minutes 39 secondes sur roo inséminations contre 7 minutes 22 secondes sur 46 inséminations) mais, en 
réalité, c'est seulement lorsque les truies montrent un comportement différent du comportement typique de l'œstrus (truies plus ou moins agitées), que l'introduction du sperme est plus rapide chez les vieilles truies que chez les jeunes truies. Dans le cas contraire, la durée de l'insémination est la même pour les nullipares et les autres, soit 6 minutes 13 secondes et 6 minutes 20 secondes. En tout cas, on enregistre une relation étroite entre la durée de l'insémination et le comportement.

Dans le lot de 133 inséminations pratiquées sur des jeunes truies dont les chaleurs étaient contrôlées, on a classé les truies suivant le moment de l'cstrus où l'insémination artificielle a été opérée (tableau 2). L'insémination est plus rapide quand elle a lieu dans les trente premières heures de l'œstrus.

TABLEAU 2

Chaleurs testies (Iruies nullipares)

\begin{tabular}{|c|c|c|}
\hline Intervalle début de Chaleur-1A & Nombre d'It & $\begin{array}{l}\text { Durée moyenne } \\
\text { de l'IA }\end{array}$ \\
\hline Infërieur à $30 \mathrm{~h} \ldots$ & 50 & $\sin 20 \mathrm{~s}$ \\
\hline Suppéricur à $30 \mathrm{~h} \ldots \ldots$ & 83 & $6 \mathrm{mnn} 19 \mathrm{~s}$ \\
\hline Total. & $1: 3: 3$ & $5 \mathrm{mn} 57 \mathrm{~s}$ \\
\hline
\end{tabular}

Ainsi, avec cette technique et ce matériel, on ne peut espérer pratiquer des inséminations trè; rapides comme dans les espèces à insémination naturelle intravaginale, mais il n'est pas impossible que la durée de la stimulation produite lors de l'insémination ait un effet sur les contractions des cornes utérines, donc sur les mouvements du sperme et peut être sur la manière dont les spermatozoïdes atteindront la jonction utéro-tubaire, leur lieu de stockage (DU MEsNr. DU Bursson et DAUZIER, I955).

Le temps nécessaire à l'insémination, compris habituellement entre 5 et ro minutes, ne constitue cependant pas un élément susceptible de faire rejeter cette technique.

\section{RÉSUITATS OBTENUS AVEC CETTE MÉTHODE}

Que peut-on attendre de cette méthode? Nous avons déjà publié un certain nombre de résultats d'expériences faites au laboratoire ou chez les éleveurs (DU Mesnil, du Buisson et DaUzier, I958-I959).

Les résultats suivants représentent ceux que l'on peut obtenir des techniques décrites dans les conditions pratiques $\left({ }^{1}\right)$.

Toutes les mises bas ont été enregistrées après une visite faite par l'inséminateur 4 à 5 mois après chaque insémination artificielle. Bien qu'environ 5 p. roo

(1) Toutes les inséminations ont été réalisées par les soins de la Coopérative Agricole d'Insémination Artificielle et d'Amélioration du Cheptel du Finistère et des Côtes du Nord. Nous remercions particulièrement le Docteur Jondet, directeur, pour sa collaboration si bienveillante et si efficace, et Messieurs TrorviliE et LEBORGNE, pour leur importante participation à ce travail. 
des truies puissent être considérées après enquête comme stériles, aucune insémination n'a été annulée; les chiffres publiés sont donc rapportés au nombre total d'inséminations pratiquées. Les inséminations ont été réalisées sur 5 cantons par des inséminateurs qui opèrent habituellement sur des vaches.

Les inséminations ont commencé en février I958; beaucoup d'éleveurs utilisaient alors l'insémination porcine pour la première fois. Aussi les résultats ont-ils été très faibles (36,8 p. I00, 32,7 p. Ioo) jusqu'au Ier avril r959 (tableau 3).

TABLEAU 3

Taux de mise bas par rapport au nombre dIA en fonction de la période

\begin{tabular}{|c|c|c|c|c|}
\hline \multicolumn{2}{|c|}{ Périodes } & Nombre d'IA & Mise bas & $\%$ \\
\hline 1958 & Depuis Février & 1142 & 420 & 36,8 \\
\hline \multirow{4}{*}{1959} & 1er Trimestre & 706 & 231 & 32,7 \\
\hline & 2eme Trimestre & 895 & 437 & 48,8 \\
\hline & $3^{\text {eme }}$ Trimestre & 696 & 348 & 50,0 \\
\hline & $4_{1}^{\mathrm{eme}}$ Trimestre & 1300 & $72 / 4$ & 55,6 \\
\hline 1960 & 1er Trimestre & $14^{\prime}+7$ & 718 & 49,6 \\
\hline \multicolumn{2}{|c|}{12 derniers mois } & 4338 & 2227 & 51,3 \\
\hline
\end{tabular}

Depuis cette date, les résultats se sont toujours améliorés et l'on a atteint 55,6 p. Ioo pour le quatrième trimestre $1959\left({ }^{1}\right)$. Mais, bien que l'on ait pris toutes précautions utiles pour conserver et utiliser le sperme et le dilueur à température invariable, on a constaté, comme l'année précédente, une chute de $6 \mathrm{p}$. Ioo durant les mois d'hiver ( $\mathrm{r}$ er trimestre I960). Nous ne savons pas si ces variations sont fortuites ou si elles sont véritablement liées à la saison, soit par action sur le sperme au moment de l'insémination, soit par une baisse de fécondité.

Sur 4338 inséminations consécutives, le pourcentage de mise bas après une insémination est de 5r,3 p. Ioo (tableau 4).

Le jour de la collecte et le lendemain le pourcentage s'élève respectivement à 53,4 p. Ioo et $5 \mathrm{I}, 2 \mathrm{p}$. Ioo (différence non significative, $X^{2}=I, 8 I$ ). Après 48 heures (J 2) le pourcentage est plus faible $(46,7 \mathrm{p}$. IO0), mais il n'est significativement différent que de celui du jour $\mathrm{O}\left(\mathrm{X}^{2}=9,47\right)$. Après 72 heures $(\mathrm{J} 3)$ et jusqu'à 6 jours, le pourcentage reste sensiblement identique : 46,7 et 45,4 p. Ioo. Mais le nombre d'inséminations à partir du $3^{\mathrm{e}}$ jour n'est pas suffisant pour en tirer une conclusion formelle.

En tout cas, l'abaissement du pouvoir fécondant du sperme au début de sa conservation est assez faible pour permettre son utilisation pendant 3 jours et éviter, dans la pratique, le gaspillage.

Les variations dans les résultats en fonction des antécédents de reproduction des truies $\left(47,5\right.$ et $\left.54, I_{7}\right)$ confirment les chiffres déjà publiés par plusieurs auteurs et

(1) Durant les $2^{\text {eme }}$ et $3^{\text {ene }}$ trimestre 1960 , on a enregistré, dans l'un des sous-centre, un taux de mise bas de 58,8 sur 1660 inséminations. 
par nous-mêmes après utilisation d'autres matériels et méthođes. Malgré les différences de taille des organes génitaux, nous utilisons la même sonde et le même volume de liquide ; il n'est pas impossible que l'on ait avantage à réduire, pour les primipares, le diamètre de la sonde et le volume du sperme dilué injecté.

\section{TABLEAU 4}

Taux de mise bas par rapport au nombre d'IA en fonction de la durée de conservation du sperme

\begin{tabular}{|c|c|c|c|c|c|c|c|c|c|}
\hline & \multicolumn{3}{|c|}{ Nullipares } & \multicolumn{3}{|c|}{ Truies ayant déjà porté } & \multicolumn{3}{|c|}{ Toutes truies } \\
\hline & IA & Mise bas & $\%$ & IA & Mise bas & $\%$ & IA & Mise bas & $\%$ \\
\hline$J_{0}(*) \ldots \ldots \ldots$ & 781 & 395 & 50,6 & 1081 & 600 & 55,5 & 1862 & 995 & 53,4 \\
\hline$J_{1} \ldots \ldots \ldots \ldots$ & 726 & 330 & 45,4 & 948 & 528 & 55,7 & 1674 & 858 & 51,2 \\
\hline$J_{2} \cdots \cdots \cdots \cdots$ & 268 & 120 & 44,8 & 394 & 189 & 47,9 & 662 & 309 & 46,7 \\
\hline$J_{3} \ldots \ldots \ldots \ldots$ & 47 & 22 & 46,8 & 60 & 28 & 46,6 & 107 & 50 & 46,7 \\
\hline $\mathrm{J}_{4}, \mathrm{~J}_{5}, \mathrm{~J}_{6} \ldots \ldots$ & 10 & 4 & 40,0 & 23 & 11 & 47,8 & 33 & 15 & 45,4 \\
\hline $\begin{array}{l}\text { Toutes durées } \\
\text { deconservation. }\end{array}$ & 1832 & 871 & 47,5 & 2506 & 1356 & 54,1 & 4338 & 2227 & 51,3 \\
\hline
\end{tabular}

$\left(^{*}\right) \mathrm{J}_{0}=$ utilisation du sperme le jour de la collecte.

\section{TABLEAU 5}

Taux de mise bas par rapport au nombre d'I $A$ en fonction du comportement des truies au moment de l'intervention

\begin{tabular}{|c|c|c|c|c|c|c|c|c|c|}
\hline \multirow[b]{2}{*}{ Comportement. } & \multicolumn{3}{|c|}{ Nullipares } & \multicolumn{3}{|c|}{ Truies ayant déjà porté } & \multicolumn{3}{|c|}{ Toutes truies } \\
\hline & IA & Mise Bas & $\%$ & IA & Mise Bas & $\%$ & IA & Mise Bas & $\%$ \\
\hline Très calme... & 351 & 199 & 56,7 & 520 & 357 & 68,6 & 871 & 556 & 63,8 \\
\hline Calme....... & 1120 & 553 & 49,4 & 1507 & 822 & 54,5 & 2627 & 1375 & 52,3 \\
\hline Agité........ & 345 & 108 & 31,3 & 461 & 167 & 36,2 & 806 & 275 & 34,1 \\
\hline $\begin{array}{l}\text { Sans renseigne- } \\
\text { ment ......... }\end{array}$ & 16 & 11 & 68,7 & 18 & 10 & 55,5 & 34 & 21 & 61,7 \\
\hline $\begin{array}{l}\text { Tous comporte- } \\
\text { ments ........ }\end{array}$ & 1832 & 871 & 47,5 & 2506 & 1356 & 54,1 & 4338 & 2227 & 51,3 \\
\hline
\end{tabular}

Enfin, le comportement de la truie au moment de l'intervention (tableau 5) joue un rôle capital dans la réussite de l'insémination $(68,6 \mathrm{p}$. Ioo chez les truies multipares très calmes) ; ceci confrme également nos résultats antérieurs obtenus cependant dans des conditions techniques très différentes (DU MESNIL, DU BUISSON et DauziEr, I959), de même que ceux de Madden (I959 et I960). 
Le nombre de porcelets par portée (tableau 6) peut être considéré comme normal, si l'on tient compte du fait que les truies inséminées sont, la plupart du temps issues de croisement. Ainsi les truies de type Normand, ont un nombre de porcelets relativement fort pour leur race et inversement les truies "Large White " qui ont souvent dans leurs ascendants des éléments de la race Normande, moins prolifique, montrent des portées en moyenne légèrement inférieures au standard de la race.

TABLEAU 6

Nombre de porcelets par portées

\begin{tabular}{|c|c|c|c|c|}
\hline \multirow{3}{*}{ Race } & \multicolumn{4}{|c|}{ Antécédents de reproduction } \\
\hline & \multicolumn{2}{|c|}{ Primipares } & \multicolumn{2}{|c|}{ Multipares } \\
\hline & $\begin{array}{l}\text { Nombre moyen } \\
\text { de porcelets }\end{array}$ & Nombre de portées & $\begin{array}{l}\text { Nombre moyen } \\
\text { de porcelets }\end{array}$ & Nombre de portées \\
\hline Normande & 6,89 & 524 & 8,72 & 885 \\
\hline Large-White & 7,41 & 245 & 9,37 & 337 \\
\hline
\end{tabular}

Il est du reste probable qu'un abaissement du nombre des spermatozoïdes par dose provoque une diminution du nombre des porcelets à la naissance (DU MESNIL. DU Bursson, Ig6r).

En conclusion, si le matériel utilisé, en polytène et en caoutchouc, présente l'inconvénient d'être difficile à nettoyer et à aseptiser, la nature-même de ce matériel, de la sonde notamment, constitue un élément de sécurité ; elle met l'inséminateur à l'abri de toute manœuvre dangereuse pour la truie; en outre, la liaison souple entre le récipient et la sonde lui épargnent la fatigue due à la poursuite de certaines truies pendant l'opération et évite à peu près complètement le reflux du sperme dilué. De plus, le transport du sperme en ampoule scellée immergée le protège contre la pollution et les variations de température. Cette méthode est donc très sure : son utilisation pendant 2 ans dans la pratique, avec contrôle des résultats à la naissance semble lui conférer un certain avantage sur les autres techniques utilisables à l'heure actuelle.

Rę̧u en novembre 1960.

\section{SUMMARY}

The Material AND teCHNiQUe OF ARTIFICIAL INSEMINATION IN THE PIG USED IN FRANCE. RESULTS OBTAINED.

The technique of insemination described here is based on the principle of flow by gravity of the semen. It has been applied to some 10000 sows in the field. The time required for an insemination depends on the cervical and uterine contractions of the individual sow. In $5^{2} \mathrm{p}$. roo of cases, the time required to inseminate $25^{\circ} \mathrm{cc}$ of semen is 4-7 minutes. The duration of the insemination is least in the first half of cestrus and in perfectly quiet sows.

The material used consists of a rigid rubber catheter $(20 \mathrm{~mm}$ diameter) surrounded at one end by an inflatable balloon ( $8 \mathrm{~cm}$ long, $35 \mathrm{~mm}$ diameter). The catheter is connected to a bottle of the 
"plasma bottle " type containing the semen through a I-meter long polythene tubing; the bottle is held $40 \mathrm{~cm}$ above the sow's back. The rubber catheter is attached to the tail by an elastic band.

The semen is prediluted, then preserved at $15^{\circ} \mathrm{C}$ in sealed vials under carbon dioxide according to the technique described earlier by DavzIes and myself. The semen is diluted a second time just before use.

The results given in the following are expressed as the percentage of checked farrowings after a single insemination -- 51.3 per 100 of $4,43^{8}$ inseminations have thus been found successful. No significant difference was found when the results recorded for semen used on the day of collection $\left(\mathrm{J}_{0}\right)$ were compared with the results obtained for semen used the day after $\left(\mathrm{J}_{1}\right)-66_{2} \mathrm{~A}$. I. carried put on day $\mathrm{J}_{2}$ gave a rate of success of $46.7 \mathrm{p}$. 100 while $107 \mathrm{~A}$. I. on day $\mathrm{J}_{3}$ also gave $46.7 \mathrm{p}$. 100 . For the subsequent days $J_{4}, J_{5}, J_{8}$ the rate of success given by a total of 33 inseminations was 45.4 p. 100.

Great differences appear in the rate of success when the behaviour of the sows at the time of insemination is taken into consideration. Thus in 87 I sows recorded as "very quiet "by the inseminator, the rate of success amounts to $63.8 \mathrm{p} .100$.

The litter sizes obtained were those considered normal for the particular breeds used, namely 8.72 on the average for the Normande and 9.37 for the Large White.

\section{RÉFÉRENCES BIBLIOGRAPHIQUES}

AaMdal J., Hogset I., I957. Artificial Insemination in Swine. J. Am. Vet. Med. Ass., 131, 59-64.

Bonadonna T., 1940. Nanuele di tecnica della Fecondazione Artiticiale degli Animali. 789 pp. Milesi et Figli éd., Milan.

GoEtze R., I949. Besamung mnd Infruchtbarkeit der Haussaügetiere. 6r 3 pp., Verlag M. u .H. Schaper, Hannover, d'après HOF. F., Die Künstliche Besamung des Schweines im Schrifttum. 'Thèse Vet. Giessen.

Ito S., Niwa T., Kudo A., 1948. On the method of injection of Semen and the results in fecondation. Zootech. Exp. Sta. Res. Bull., 55, 57-74.

Kvas:iski A.V., I959. Méthode fractionnée d'Insémination Artificielle des Truies. Colloque Reprod. Insem. Art. Porc, Ann. Zootech. Supp., 1959, 43-58.

Kvasiski A.V., Konyukova L. A., I958. Méthodes d'Insêmination Artificielle pour les Truies. Vest. Selskokhoz. Nauki, 3, 89-96.

Liebenberg O., 1952. Die Besamung der Hausliere, insbes. des Rindes, Neumamn Ed. Radebeul et Berlin, so pp., d'après HÖF. F., I956, Die Künstliche Besaniung des Schweines im Schrifttum, Thèse Vet. Giessen.

MADDEN D H.I. I959 Field experience of Pig A.I. in Hampshire, U.K. Colloque Reprod. Insem. Art. porc. Ann. Zootech. Supp. I959, 59-67.

Madden D.H.L. ig6o, Pig A.I. Vel s Rec. 72885.886.

DU Mesvil DU Buisson F., I96r. Utilisation du $\mathrm{CO}^{2}$ dans l'insémination porcine. IV" Congrès Interh. Reprod. anim. La Haye.

DU Mesnil du Buisson F., Dauzier L., I955. Distribution et résorption du sperme dans le tractus génital de la Truie. Survie des spcrmatozoïdes. Ann. Endocrinol., 16, 4I 3-422.

DU Mesnil DU BuIsson F., DAUzIER L., I959. Amélioration des techniques de conscrvation de la semence de Verrat par dissolution à saturation d'anhydride carbonique dans le milieu de conservation, et conditions de leur utilisation pratique. Colloque Reprod. Insent. Art. Port, Ann. Zootech., Supp. 1959, 81-96.

Milovaxov V.K., I934. Artificial Insemination in Livestock. Anim. Breed. Abst., 2, 403.

NiwA T., 1948. Artificial Insemination with Swine in Japan. Nat. Instit. Agric. Sci. Chiba, I fasc., I 3 pp,

POLGE C., I956. The development of an artificial Insenination Service for Pigs. Anim. Breed. Abst., 24, $209-217$.

Vax Demark M. L., Sharma U.D., I957. Preliminary fertility results from the preservation of Bovine Semen at room temperature. J. Dairy Sci., 40, 438-439. 